\title{
Immunohistochemical Identification of a Malignant Tumor of the Heart.
}

\section{S. Siew* and N.C. Caliman**}

*Michigan State University, East Fee Hall, A-634, East Lansing, MI 48824

**Ingham Regional Medical Center, 401 W. Greenlawn Ave., Lansing, MI 48910

We report the case of a 54-year-old woman, who presented with shortness of breath and fatigue. Chest x-ray showed the presence of an increased amount of fluid, around the heart, in the pericardial sac. Cardiac catheterization was performed and demonstrated encroachment by a mass upon the cavity of the right ventricle. Biopsies were taken, through a thoracotomy, of the right ventricle and the parietal pericardium. The right ventricular biopsies consisted of multiple fragments of gray-brown tissue, measuring overall, $1.2 \times 1.0 \times 0.5 \mathrm{~cm}$. The pericardial biopsy consisted of a piece of yellow-brown tissue, covered with fat.

Histopathologic examination showed the presence of an infiltration of sheets of malignant cells in a fibrovascular stroma. No normal myocardial tissue could be identified. The cells were polygonal and the nuclei, pleomorphic. There was a high nucleocytoplasmic ratio, in most instances. The cytoplasm was clear and there was little cytoplasmic detail, apart from occasional fine strands which could be identified in the cytoplasm (Fig. 1). Foci of hemorrhage were observed. The pericardial tissue showed the presence of chronic pericarditis. There was no evidence of malignant infiltration.

In the involvement of the heart by malignant tumors, metastatic tumors are 16-40 times more common than primary tumors which originate in the heart. $85 \%$ of these involve the pericardium. The lack of differentiating features in the malignant cells opened a wide field of possibilities, including malignant lymphomas and other small round cell tumors such as Ewing's sarcoma and rhabdomyosarcoma as well as tumors of epithelial origin.

An extensive Immunohistochemical work-up was undertaken:

Malignant lymphoma: CD 20 to identify a B cell lymphoma; CD 30 an anaplastic large cell lymphoma; CD 45 - leukocyte common antigen, present on all leukocytes. All of the control tests were positive and the biopsy tissue was negative. Epithelial neoplasia: Epithelial membrane antigen (EMA); AE1 and AE3 to identify cytokeratin. All of the control tests were positive and the biopsy tissue was negative. The Alcian blue-Periodic acid Schiff technique was applied in order to identify Ewing's sarcoma and it was negative. The myoglobin 1 test was positive (Fig. 2). This is consistent with the diagnosis of a rhabdomyosarcoma. Re-examining the histopathologic material, greater attention could be paid to the fine strands in the clear cytoplasm. These represent scattered myofilaments in a primitive myoblast.

Primary rhabdomyosarcoma of the heart is a rare tumor. There were only 26 cases in the collection of the Cardiovascular Division of the Armed Forces Institute of Pathology. The cases showed a very wide range in age, but, were less common in children. A slightly higher incidence has been reported in men. The prognosis is poor as the majority of patients die within a year. Survival for 3 years has resulted after excision of the tumor followed by combined radiation therapy and chemotherapy. 


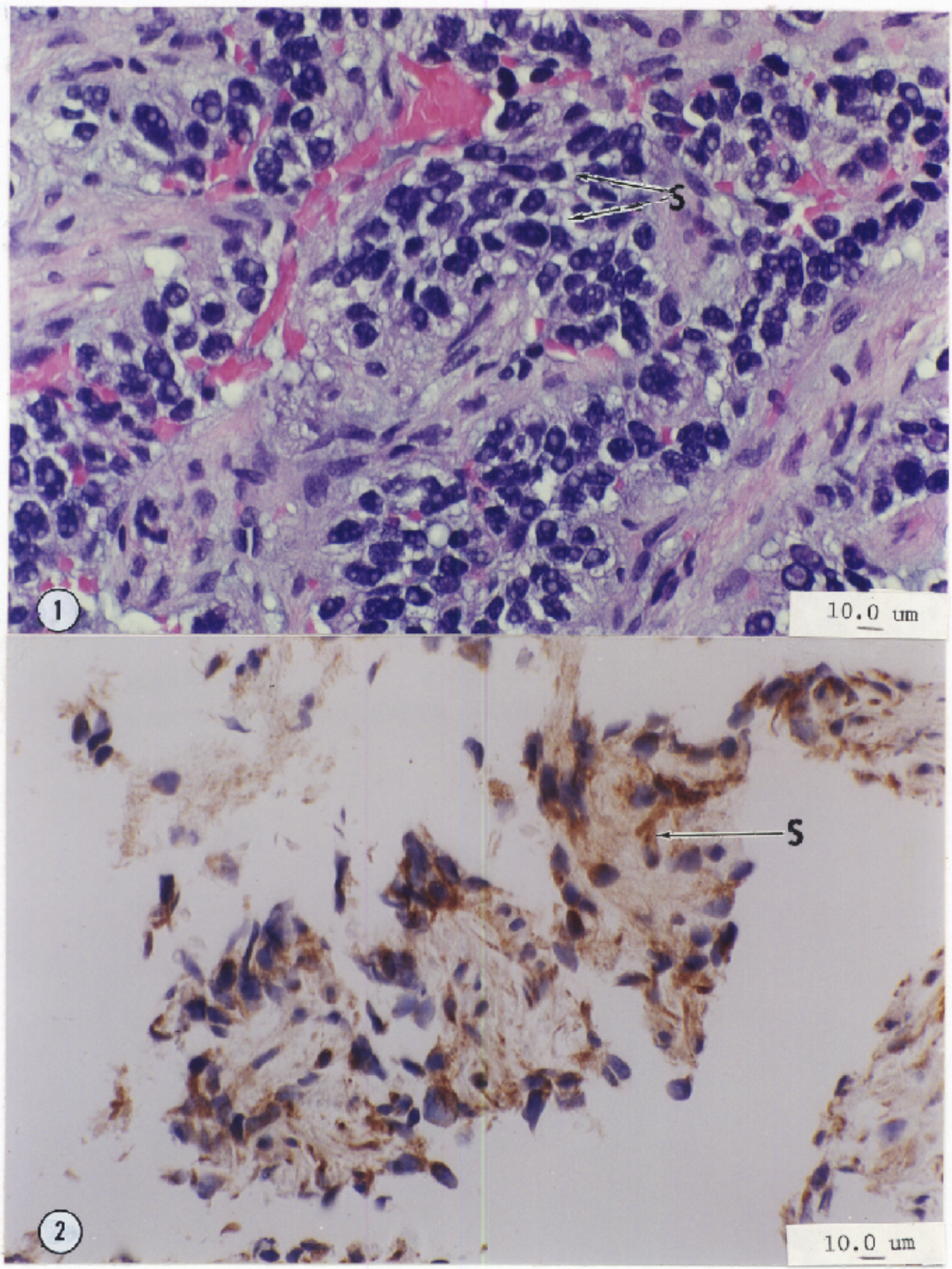

Fig. 1. Sheets of malignant cells in a fibrovascular stroma. S - fine cytoplasmic strands Fig. 2. Immunohistochemistry Myoglobin 1. Positive reaction by cytoplasmic strands 